\title{
Technology and Teenagers: No Time to Sleep?
}

\section{Siobhán O’Neill}

\author{
School of Applied Psychology, UCC
}

Sleep is that golden chain that ties health and our bodies together. (Thomas Dekker)

\section{The "lazy teenager"}

It's 7am on Tuesday morning and your alarm is blaring beside you. You feel groggy and disoriented and you know you have to get up but you hit the snooze button instead, unable to motivate yourself to get up. Twenty minutes later your mother comes into your room to wake you for school, you're going to be late again. You struggle out of bed to the words, "You're so lazy! What time did you go to sleep last night? You need to go to bed earlier." Once at school you go through the motions of classes, breaks and socialising. However, as the day goes on you find yourself getting sleepier and sleepier, unable to fully concentrate on the teachers or even your friends. You wish it were Saturday already so you can get that much needed lie in. But Saturday feels like such a long way away with a week full of school, homework, football training and your part-time job to get though first. Maybe your Mum is right, you're just lazy and need to go to sleep early tonight.

We've all heard the term "teenagers are lazy", we've probably even been called lazy ourselves at some point during our adolescence. There is a stereotypical notion that teenagers will stay in bed all day and sleep if they were left to because they are lazy. What if I told you that this stereotype is misleading and unfair? What if I told you that teenagers can't help wanting to stay in bed until mid-morning or later? Well it's true.

During puberty the body goes through biological changes that affect the sleep hormone melatonin, causing it to be secreted later in the evening. Melatonin determines sleep onset and is regulated by light. As the day goes on, light starts to fade and melatonin begins to be produced by the body, it reaches peak secretion levels in the middle of the night and then declines to low levels in the day. (See Figure 1 below). In adolescence, melatonin is secreted later meaning that sleep onset shifts to a more evening preference and a later bedtime, and so teenagers are biologically predisposed to stay awake later at night.

Although adolescents go to bed later at night due to these biological changes, their daily schedules are still dictated by societal norms. For instance school start times are scheduled for the early morning and in many instances second level schools start earlier than primary schools. These early mornings conflict heavily with the biological clocks of teenagers and 


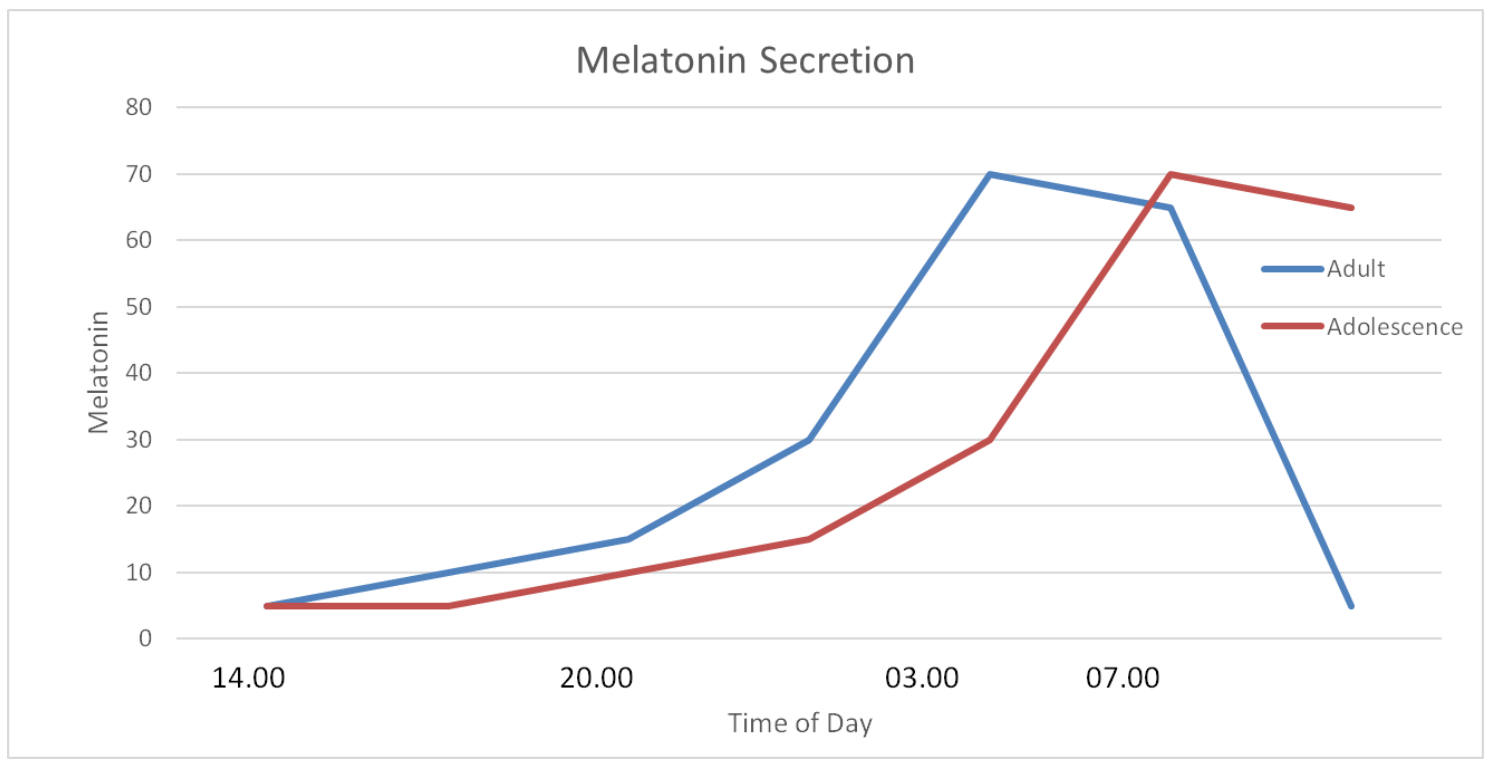

Figure 1: Daily Melatonin Secretion Levels. Source: Siobhan O’Neill

can cause excessive daytime sleepiness and the need to catch up on sleep debt at the weekends.

\section{Stress during adolescence}

As sleep patterns alter during adolescence, similarly experiences of stress can change during this developmental period. Adolescence is marked by novel experiences and biological changes in the body and these can increase the chances of stressful experiences occurring.

During the stress response a hormone called cortisol is secreted, this hormone readies the body for action by increasing heart rate and supplying large muscles with energy. This is an adaptive response to stress, however prolonged or chronic secretion of cortisol can have many adverse effects on the body and health including increasing the risk for hypertension, obesity and memory problems. Previously it was thought that the effects of stress-mediated cortisol did not emerge until adulthood, but there is accumulating evidence that these effects are present in adolescence.

\section{Sleep, stress, and health}

Poor sleep can have many deleterious effects on health and wellbeing. While asleep, our bodies maintain our physical health and support brain functioning, and during childhood and adolescence sleep also supports development. Insufficient sleep and poor quality sleep can have a large impact on your physical health, and has been linked to chronic illnesses such as: diabetes, cardiovascular disease, kidney disease, and obesity. 
Table 1: Technology Use at Night, Sleep in America Poll 2011

\begin{tabular}{|l|l|l|}
\hline Technology Type & $\begin{array}{l}13-18 \text { Year } \\
\text { Olds }\end{array}$ & $19-29$ Year Olds \\
\hline Phones & $72 \%$ & $67 \%$ \\
\hline Computers/Laptops & $60 \%$ & $60 \%$ \\
\hline Music Device & $64 \%$ & $43 \%$ \\
\hline Television & $54 \%$ & $59 \%$ \\
\hline Video Games & $23 \%$ & $18 \%$ \\
\hline
\end{tabular}

1. Poor sleep can also have an impact on cognitive function and performance. A lack of sufficient sleep can cause short-term memory problems, work performance issues, poor academic achievement, mood swings, behavioural problems and daytime sleepiness.

2. Similarly stress has been linked to many poor health outcomes including poor sleep, cardiovascular disease, and depression. Evidence from studies of adults suggests a strong role of early life stress on the risk of obesity. Adolescents with obesity have a higher likelihood of contracting these serious life-threatening in adulthood and this may be the result of too much stress or too little sleep, or more likely the interaction of both of these factors.

\section{Sleep and technology}

Technology use has become a normal part of everyday life for many, especially young people. Figures from the Sleep in America Poll (2011) indicate that the 13-18 year old age group use far more technology than any other, and a significant percentage of people aged 13-29 use multiple devices in bed during the hour before falling asleep (Table 1). This is very problematic in terms of preparing the body for sufficient sleep quality and duration. Adolescents are delaying their sleep onset by engaging with devices that stimulate the minds and arouse their emotions. Not only that, these devices are also affecting their natural melatonin secretion pattern. Blue light is emitted from devices such as phones, laptops and tablets, and this blue light has been found to delay melatonin secretion and therefore sleep onset by up to two hours when used before bedtime or whilst in bed in an adult population.

\section{My research}

My first study, The CLASS Study (Changing Lives: Adolescent Stress and Sleep), is a longitudinal project investigating the effects of stress, sleep, and technology use on health in adolescents in Ireland. In particular the effects of poor sleep and prolonged, chronic 
stress on obesity will be examined. The central research questions for this study are as follows:

Does technology use before bed delay sleep onset in adolescence?

Does stress and higher levels of cortisol contribute to the onset of poor health outcomes, including obesity, in adolescence?

Does poor sleep contribute to the onset of obesity during adolescence?

Is there an interaction effect between sleep, technology use, and stress on obesity during adolescence?

Participants take part in the study for a 12 month period, with data collection occurring three times in total at 6 month intervals (0, 6 and 12 months). The participants are aged between 12 and 25, and include both second and third level students.

My study incorporates both standardised questionnaires on sleep, stress and health, as well as daily diaries of stressful experiences and food intake. Participants are also asked a series of questions about their use of technology, both during the hours before bed and their levels of engagement with technology on a daily and weekly basis. Participants also provide saliva and blood samples to measure their stress hormone, cortisol, and a number of other hormones linked to chronic stress.

At each of the three sessions participants are seen in their school where they fill in the survey and provide height and weight measurements, and then for four days following that session (Saturday-Tuesday) they fill in the daily diaries and provide a number of saliva samples to capture their individual daily cortisol patterns. This research is currently on-going.

My second study is an online survey examining the factors that contribute to poor sleep. As mentioned above technology use has been found to delay sleep onset due to the suppression of melatonin by blue-light emissions from devices. However, very little research has examined the effects of the emotional arousal caused by technology use and its effects on sleep onset. Is delayed sleep onset caused purely by the biological effects of blue light, or does level of emotional arousal also play a part?

The main aim of this survey is to investigate the impact technology use, reading, and listening to music has on sleep onset and also to investigate the impact that the emotional arousal of these activities has on sleep onset. The secondary aim of the study is to investigate how pets, children and environmental noise impact on sleep quality and duration, in order to more fully understand the complexities of contributing factors to poor sleep. This study will recruit participants from around the world and will investigate cross-cultural differences in sleep quality. 


\section{Research impact}

It is clear that poor sleep and stress can have a deleterious effect on health. The CLASS Study is the first study to investigate the combined effects of poor sleep and stress on obesity in an Irish adolescent population. There is a strong body of research investigating the effects of stress on an adult population but there is significantly less investigating stress in adolescence. This study has the potential to impact on public best practice policy for sleep and health guidelines in relation to obesity. This research could also inform interventions that would potentially lessen the likelihood of adolescent and long-term obesity in an Irish population.

The online study may provide a more cohesive understanding about the factors which contribute to delayed sleep onset, poor sleep quality, and poor sleep duration. It will also determine the impact of emotional arousal of bedtime activities on sleep hygiene. It will also potentially add to the best practice literature on good sleep hygiene. The online survey may also provide information for the development of a new standardised questionnaire on sleep hygiene.

Future research needs to examine the effects and interactions of melatonin secretion and emotional arousal on sleep onset in adolescence in order to fully investigate the impact of technology use on sleep. As obesity during adolescence has been linked to long-term obesity and chronic illness, further investigation is needed on the causes of obesity at this time and during childhood in an Irish population.

I would like to thank my supervisor Dr. Samantha Dockray, for her support and advice. I would also like to thank the participants who have taken part in The CLASS Study. Funding for this work was provided by the Irish Research Council. 